\title{
The State of Wetlands of the Manipur Valley in Northeast India in View of Changing Time
}

\author{
Premananda Laishram, Kshetrimayum K.S. \\ Department of Earth Science, Assam University, Silchar - 788011, India \\ *Corresponding author: drkrishnakanta@gmail.com
}

\begin{abstract}
The Manipur valley is located as an intramontane basin filled with alluviums of fluvio-lacustrine origin of Quaternary age in the Indo-Myanmar Range of Northeast India. The valley is confined between $24^{\circ} 16^{\prime}$ and $25^{\circ} 2^{\prime}$ North latitudes and $93^{\circ} 41^{\prime}$ and $94^{\circ} 9^{\prime}$ East longitudes covering an area of $\sim 1920 \mathrm{~km}^{2}$ with a population of more than 2 million people. This valley is mainly occupied by wetlands like ponds, swamps, paleochannels, lakes, agricultural fields and flood plains. Historically, these wetlands have been emotionally relating to cultural and ritual activities, fortification and recreational activities since the beginning of the Manipuri culture. To assess the quality of these wetland waters, several hydrochemical parameters like $\mathrm{pH}$, Temperature, TDS, EC, ORP, $\mathrm{HCO}_{3}, \mathrm{CO}_{3}, \mathrm{SO}_{4}, \mathrm{Cl}, \mathrm{Na}$, $\mathrm{K}, \mathrm{Mg}, \mathrm{Ca}, \mathrm{PO}_{4}, \mathrm{~F}, \mathrm{H}_{4} \mathrm{SiO}_{4}, \mathrm{Ti}, \mathrm{V}, \mathrm{Cr}, \mathrm{Cu}, \mathrm{Ge}, \mathrm{As}, \mathrm{Rb}, \mathrm{Sr}, \mathrm{Nb}, \mathrm{Mo}, \mathrm{Hf}, \mathrm{Ta}, \mathrm{W}$ were determined. The analysis suggests that the water is fresh and suitable for human consumption as well as and flora and fauna adaptation. Presently, the wetlands are used for water supplies for domestic, irrigation, industrial purposes and fish production. The rapid urbanization, massive increase in population, local climate change cause significant diminishing and shrinkage of these wetlands and thus creating a cultural gap as the ritual activities around these surface water bodies are no more practiced. Further, mismanagement and negligence on the treatment of domestic solid waste deteriorate the quality. Artificial eutrophication coupled with climate change as it reduces oxygen level and increases acidic level in the wetland waters further worsen the quality leading to the extinction of some fish species from these waters. Therefore, it is high time to avoid demolishing these wetlands and rejuvenate them to maintain the age old socio-cultural relationship it bears.
\end{abstract}

Keywords: wetlands, kingdom, society, urbanization, eutrophication, Manipur valley

Cite This Article: Premananda Laishram, and Kshetrimayum K.S., "The State of Wetlands of the Manipur Valley in Northeast India in View of Changing Time.” American Journal of Water Resources, vol. 6, no. 1 (2018): 9-14. doi: 10.12691/ajwr-6-1-2.

\section{Introduction}

In Manipuri society, the role of wetland in the form of a pond, swamps, lakes, paleochannels etc. is manifold and makes an absolute contribution to daily life. Yet, they have been a victim of serious scientific and social negligence. Recent studies based on Quick Bird satellite image of $61 \mathrm{~cm}$ resolution prepared at 1:4000 scale [1] revealed that Manipur valley occupies an area of about $1920 \mathrm{~km}^{2}$ in which natural wetlands cover $61.2 \mathrm{~km}^{2}$. Out of this wetlands area, ponds which are very common to people living in this valley contribute an area of $6.8 \mathrm{~km}^{2}$ which turns out to be around $0.35 \%$ of the total area of the valley. The local term of pond, 'pukhri' is believed to be borrowed from Bengali word called 'pukur' which means pond. The term 'pond' is a variant form of the word pound, meaning a confining enclosure. Scientific definition of a pond is still not universally defined. However, the international Ramsar wetland convention sets the upper limit for pond size as 8 ha. British charity pond conservation have defined a pond to be a man-made or natural water body which is between 1 ha and 2 ha in area which holds water for four months of the year or more.
Other European biologists have set the upper size limit at 5 ha. By and large, a pond implies a quite small body of water, generally smaller than one would require a boat to cross or a body of water where even its deepest areas are reached by sunlight or where a human can walk across the entire body of water without being submerged [2]. It is believed that the use of wetlands particularly ponds by the people of this valley dated back to the 1st Century during King of Manipur, Nongdon Lairen Pakhangba (33-154 AD). 'Cheitharol Kumbada' (the royal chronicle of Kangleipak) refers that ancient rituals were performed on the bank of ponds. A famous pond called Nungjen Pukhri located inside the Kangla palace of Manipur was believed to be abode of Lord Pakhangba of Manipur. Other names of the ponds in Kangla Palace like Koubru Pukhri, Chingkhei Nungjeng, Manung Nungjeng and Lai Pukhri are still considered as sacred where religious rituals were performed. The ponds were used for recreation and adornment purposes since the early times. King Khunjaoba (1632-1666 AD) excavated a moat which locally called 'Thangapat' on the western side of the Kangla Fort located in the Manipur valley for its fortification and beautification. Ponds were also use as venue for performing Ras Lila which is a cultural activity during King Bhagyachandra (Meidingu Chingthang 
Khomba) time (1749-1798 AD). Ningthem Pukhri which is considered as the largest pond $\left(4031 \mathrm{~m}^{2}\right)$ in the valley was excavated by King Garib Niwaj (Pamheiba) in 1726 [3]. This article attempts to throw some lights on the present state of such wetlands from the Manipuri socio-cultural perspective so as to help in rejuvenating and developing of wetlands in the Manipur valley.

\section{Study Area}

The Manipur valley is locate at $\sim 780 \mathrm{~m}$ elevation above mean sea level in the Indo-Myanmar Range (Figure 1). The valley is a small pull-apart (transitional) basin in the later phase of the Indo-Myanmar orogeny [4]. The valley is mainly occupied by alluviums of fluvio-lacustrine origin of Quaternary age. The alluviums are mainly composed of carbonaceous clay, silt and sand and exclusively confined within an anticlinorium which characterized by several anticlines and synclines in the Disang Group [5]. The average thickness of the alluvium as per drilling data is around $\sim 200 \mathrm{~m}$ and they abut against the surrounding Tertiary hills with distinct fan characters. The Imphal river and its tributaries like the Iril, the Thoubal and the Nambul rivers flow almost in N-S direction in the valley. Hydrogeologically, the valley consists of alluviums of Quaternary formation and semi-consolidated formations of shale, siltstones and conglomerate of Tertiary group. Groundwater is restricted to weathered residium of semiconsolidated rocks and intergranular pore spaces of alluvial deposits under water table to confined conditions. Generally, groundwater at shallow levels occur at $\sim 3 \mathrm{~m}$ to $\sim 4 \mathrm{~m}$ below ground level (bgl) under unconfined conditions while in deeper levels it occurs at $\sim 7 \mathrm{~m}$ bgl to $\sim 15 \mathrm{~m} \mathrm{bgl}$ [6]. Most of the borewells tapped the top unconfined aquifers. The average transmissivity and hydraulic conductivity ranges between 4.30 and $89 \mathrm{~m}^{2}$ /day and 0.67 $\mathrm{m} /$ day to $16 \mathrm{~m} /$ day respectively. The water level fluctuation of pre and post monsoon is about $12 \mathrm{~m}$ [7].

\section{Materials and Methodology}

38 surface water samples were collected from different locations of wetlands located in the Manipur valley. The wetlands include ponds, swamps, paleochannels and lakes. Basic physico-chemical parameters like temperature $(T)$, $\mathrm{pH}$, total dissolved solids (TDS) and oxidation reduction potential (ORP) were measured using filed potable kit, Model No. HI 9811-5, Hanna at the time of sample collection. Samples were filtered with a hand-held syringe using $0.45 \mathrm{~mm}$ membrane filters and collected in air proof new $200 \mathrm{ml}$ polyethylene bottles. The water samples were acidified using $2 \mathrm{M} \mathrm{HNO}_{3}$ acid to $\mathrm{pH} 2$ for preservation for cations and heavy metals tests. Analytical procedure and testing for $\mathrm{SO}_{4}, \mathrm{PO}_{4}, \mathrm{H}_{4} \mathrm{SiO}_{4}, \mathrm{~F}, \mathrm{NO}_{3}$ and heavy metals like Ti, V, Cr, Cu, Ge, As, Rb, Sr, Nb, Mo, Hf, Ta and $\mathrm{W}$ were determined by spectrophotometer ICP (MS) at Pondicherry University, India. $\mathrm{Na}$ and $\mathrm{K}$ were determined by ICP (AES) in the same laboratory. $\mathrm{CO}_{3}$, $\mathrm{HCO}_{3}, \mathrm{Cl}$, Ca and $\mathrm{Mg}$ were determined at Indian Institute of Technology, Bombay, India. To collect the detail information of wetlands like ponds, Google image 2017 Digital Globe was used.

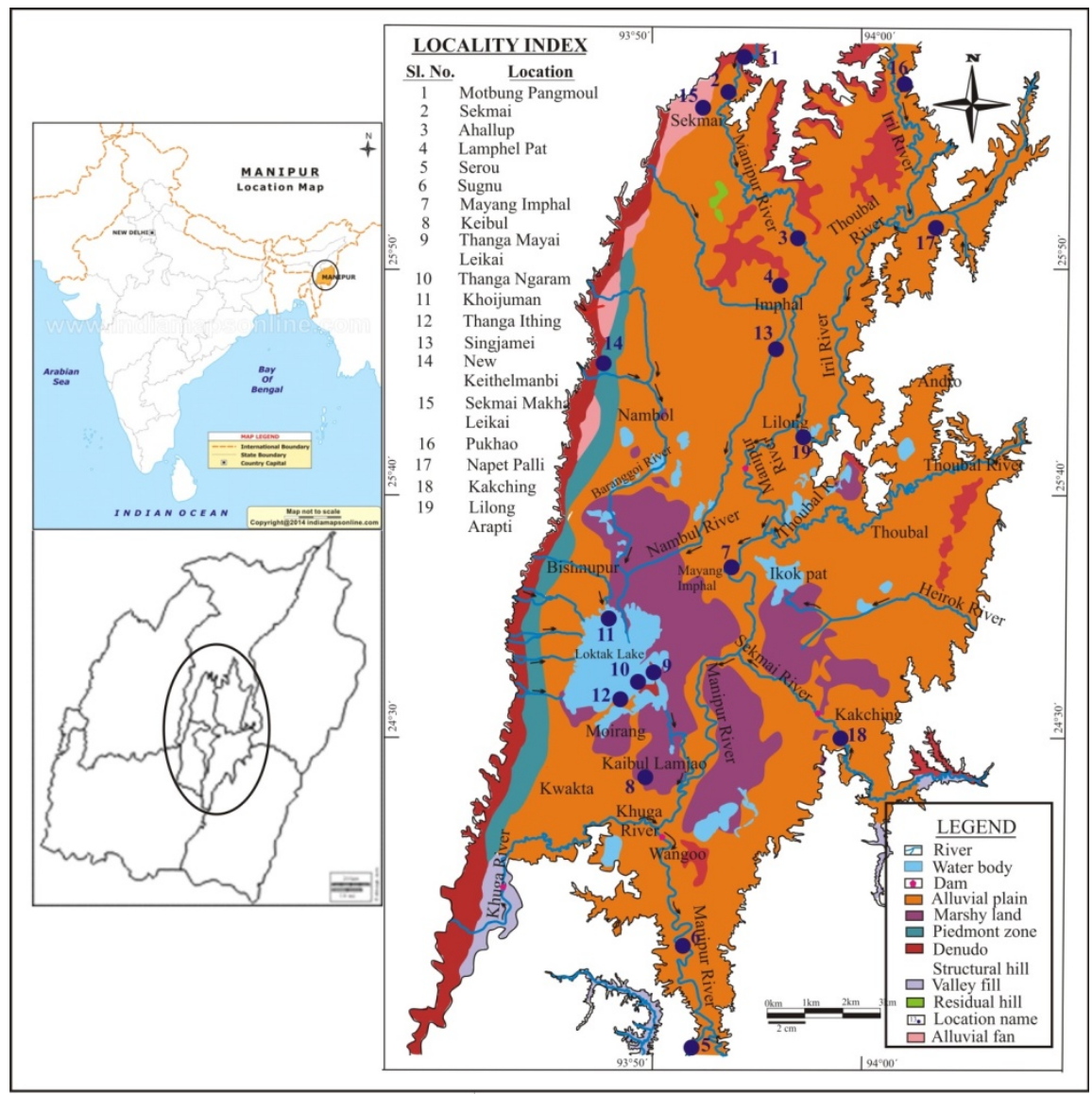

Figure 1. Map of Manipur valley showing the sampling sites in the wetlands (modified after SOI, Toposheet No.83G, H, L, K) 


\subsection{Elementary Geochemistry}

The results of hydrochemical analysis and summary of statistical data is shown in Table 1 \& Table 2. Generally, $\mathrm{pH}$ values indicate the neutral type of water (7.3) in these wetlands though the water becomes slightly alkaline (9.3) to some of the wetlands. TDS values ( $85 \mathrm{mg} / \mathrm{l})$ also show fresh water type. However, ORP values show negative $(-29 \mathrm{mV})$ indicating significant dissolution of ions under anoxic condition. Generally, wetlands have low $\mathrm{SO}_{4}$ concentration $(8 \mathrm{mg} / \mathrm{l})$ indicating reduced environment.
Concentrations of major ions like $\mathrm{Ca}, \mathrm{Mg}, \mathrm{K}, \mathrm{HCO}, \mathrm{CO}_{3}$ are found normal as their concentrations fall within the permissible limit and hence indicating fresh water. The concentrations of metals like Ti, V, Cr, Ge, As, Rb, $\mathrm{Sr}, \mathrm{Nb}, \mathrm{Mo}, \mathrm{Hf}, \mathrm{Ta}, \mathrm{W}$ show significant elevations than WHO recommended limits. Sr and $\mathrm{Cr}$ are found highly elevated in these waters. The source of theses metal elevations may be from geogenic weathering of the Manipur Ophiolite Belt which exists in the northeastern part of the valley and industrial and domestic waste disposal at places.

Table 1. Detail information and hydrochemical parameters of water samples collected from wetlands of the Manipur valley, NE India

\begin{tabular}{|c|c|c|c|c|c|c|c|c|c|c|c|c|c|}
\hline Sample ID & \multicolumn{3}{|c|}{ Location } & Type of water & \multicolumn{3}{|c|}{ Geographical Coordinate } & Elevation (m) & $\mathrm{T}\left({ }^{\circ} \mathrm{C}\right)$ & \multicolumn{2}{|c|}{ TDS (ppm) } & \multirow{2}{*}{$\begin{array}{c}\mathbf{p H} \\
6.3\end{array}$} & ORP (mV) \\
\hline 1RW & \multicolumn{3}{|c|}{ Motbung Pangmoul } & River water & \multicolumn{3}{|c|}{ N2500.459' E 93⒌747' } & 874 & 18 & 6 & & & 26 \\
\hline 2RW & Sekan & & & River water & $\mathrm{N} 24^{\circ} 5$ & 384' E93 & $53.116^{\prime}$ & 736 & 16 & 6 & & 7.1 & -19 \\
\hline 3RW & Ahall & & & River water & $\mathrm{N} 24^{\circ} 5$ & 300' E93 & $56.841^{\prime}$ & 745 & 16 & 7 & & 7.8 & -57 \\
\hline 4SW & Lamp & el Pat & & Swamp water & $\mathrm{N} 24^{\circ} 4$ & 943' E93 & 54.963' & 724 & 20 & 1. & & 7.1 & -18 \\
\hline 5RW & Serou & & & River water & $\mathrm{N} 24^{\circ} 1$ & 231' E93 & $52.447^{\prime}$ & 714 & 20 & 6 & & 7.5 & -36 \\
\hline $6 \mathrm{RW}$ & Sugnu & & & River water & $\mathrm{N} 24^{\circ} 1$ & 802' E93 & $52.303 '$ & 734 & 2 & 8 & & 7.7 & -50 \\
\hline 7RW & Maya & g Imphal & & River water & $\mathrm{N} 24^{\circ} 3$ & .997 E93 & $53.375^{\prime}$ & 779 & 23 & 2 & & 6.9 & -11 \\
\hline 8LW & Keibu & & & Lake water & $\mathrm{N} 24^{\circ} 2$ & 356' E93 & 49.044' & 746 & 22 & 8 & & 6.3 & 26 \\
\hline 9LW & Thang & Mayai Le & & Lake water & $\mathrm{N} 24^{\circ} 3$ & 933' E93 & $50.236^{\prime}$ & 767 & 20 & 6 & & 7.2 & -24 \\
\hline $10 \mathrm{LW}$ & Thang & Ngaram & & Lake water & $\mathrm{N} 24^{\circ} 3$ & 413' E93 & 48.775' & 766 & 18 & 9 & & 7.2 & -24 \\
\hline $11 \mathrm{LW}$ & Khoij & man & & Lake water & $\mathrm{N} 24^{\circ} 3$ & 993' Е 93 & 47.023' & 717 & 19 & 9 & & 6.2 & 35 \\
\hline $12 \mathrm{LW}$ & Thang & Ithing & & Lake water & $\mathrm{N} 24^{\circ} 3$ & 424' E93 & 48.778' & 755 & 2 & 6 & & 7.3 & -30 \\
\hline 13RW & Singja & & & River water & $\mathrm{N} 24^{\circ} 4$ & 974 ' E93 & $56.380^{\prime}$ & 774 & 19 & 6 & & 7.4 & -37 \\
\hline $14 \mathrm{PW}$ & New I & eithelmanb & & Pond water & $\mathrm{N} 24^{\circ} 4$ & 368' E93 & 47.553' & 787 & 17 & 5 & & 7.0 & -14 \\
\hline 15PW & Sekm & Makha Le & kai & Pond water & $\mathrm{N} 24^{\circ} 5$ & 297' E93 & $52.755^{\prime}$ & 785 & 20 & 1 & & 9.3 & -129 \\
\hline $16 \mathrm{PW}$ & Pukha & & & Pond water & $\mathrm{N} 25^{\circ} 0$ & 243' E94 & 01.794' & 785 & 2 & 1 & & 7.6 & -43 \\
\hline 17PW & Napet & Palli & & Pond water & $\mathrm{N} 24^{\circ} 5$ & 836' E94 & 04.049' & 788 & 19 & 5 & & 7.9 & -63 \\
\hline 18RW & Kakcl & & & River water & $\mathrm{N} 24^{\circ} 2$ & 960' E93 & 58.839' & 794 & 19 & 6 & & 7.7 & -54 \\
\hline 19RW & Lilong & Arapti & & River water & $\mathrm{N} 24^{\circ} 4$ & 511' E93 & $57.324^{\prime}$ & 761 & 1 & 1 & & 7.4 & -37 \\
\hline $\begin{array}{c}\text { Sample } \\
\text { ID }\end{array}$ & $\begin{array}{c}\mathrm{CO}_{3}^{-} \\
(\mathrm{mg} / \mathrm{L})\end{array}$ & $\begin{array}{l}\mathrm{HCO}_{3}{ }^{-} \\
(\mathrm{mg} / \mathrm{L})\end{array}$ & $\begin{array}{c}\mathrm{Cl}^{-} \\
(\mathrm{mg} / \mathrm{L})\end{array}$ & $\begin{array}{c}\mathrm{SO}_{4}{ }^{2-} \\
(\mathrm{mg} / \mathrm{L})\end{array}$ & $\begin{array}{c}\mathrm{PO}_{4}{ }^{3-} \\
(\mathrm{mg} / \mathrm{L})\end{array}$ & $\begin{array}{c}\mathrm{NO}_{3}^{-} \\
(\mathrm{mg} / \mathrm{L})\end{array}$ & $\begin{array}{c}\mathrm{SiO}_{2} \\
(\mathrm{mg} / \mathrm{L})\end{array}$ & $\begin{array}{c}F^{-} \\
(\mathrm{mg} / \mathrm{L})\end{array}$ & $\begin{array}{c}\mathrm{Ca}^{2+} \\
(\mathrm{mg} / \mathrm{L})\end{array}$ & $\begin{array}{c}\mathrm{Mg}^{2+} \\
(\mathrm{mg} / \mathrm{L})\end{array}$ & $\begin{array}{c}\mathrm{Na}^{+} \\
(\mathrm{ppm})\end{array}$ & $\begin{array}{c}\mathrm{K}^{+} \\
(\mathrm{ppm})\end{array}$ & $\begin{array}{c}\mathbf{T i} \\
(\mathbf{p p m})\end{array}$ \\
\hline 1RW & 24 & 36.6 & 70.9 & 6.8 & 1.1 & 3.6 & 192.7 & 0.8 & 8 & 24.4 & 11.5 & 0.8 & 0.8 \\
\hline 2RW & 16 & 36.6 & 141.8 & 8.1 & 1.5 & 3.7 & 226.7 & 0.9 & 16 & 2.4 & 8.6 & 1.2 & 0.7 \\
\hline 3RW & 24 & 48.8 & 70.9 & 11.1 & 1.2 & 3.5 & 264.4 & 0.8 & 148 & 74.4 & 13.1 & 1.0 & 1.2 \\
\hline 4SW & 28 & 48.8 & 106.4 & 8.0 & 1.1 & 4.5 & 5.0 & 0.8 & 20 & 7.2 & 23.8 & 5.4 & 1.1 \\
\hline $5 \mathrm{RW}$ & 24 & 12.2 & 70.9 & 3.9 & 2.6 & 1.5 & 111.7 & 0.8 & 100 & 40.8 & 9.1 & 0.6 & 0.7 \\
\hline $6 \mathrm{RW}$ & 28 & 48.8 & 70.9 & 5.7 & 1.7 & 6.0 & 148.1 & 1.0 & 16 & 2.4 & 16.6 & 1.3 & 1.5 \\
\hline 7RW & 24 & 61 & 70.9 & 46.8 & 3.3 & 4.8 & 120.2 & 0.8 & 16 & 7.2 & 14.7 & 1.6 & 0.8 \\
\hline 8LW & 24 & 48.8 & 88.6 & 4.3 & 2.8 & 1.0 & 108.8 & 0.8 & 8 & 2.4 & 9.9 & 1.8 & 1.4 \\
\hline 9LW & 36 & 12.2 & 53.2 & 0.7 & 2.3 & 5.6 & 19.5 & 0.8 & 136 & 69.6 & 11.5 & 1.4 & 0.3 \\
\hline $10 \mathrm{LW}$ & 36 & 48.8 & 70.9 & 0.2 & 2.0 & 6.5 & 18.9 & 0.8 & 12 & 4.8 & 9.1 & 1.4 & 0.3 \\
\hline $11 \mathrm{LW}$ & 28 & 61 & 53.2 & 0.1 & 2.9 & 1.3 & 64 & 0.82 & 12 & 2.4 & 15.3 & 4.9 & 7.9 \\
\hline $12 \mathrm{LW}$ & 24 & 85.4 & 35.5 & 0.2 & 2.2 & 7.0 & 11.1 & 0.8 & 48 & 16.8 & 9.7 & 1.5 & 0.4 \\
\hline 13RW & 60 & 85.4 & 88.6 & 11.6 & 3.0 & 5.3 & 239.8 & 0.8 & 12 & 38.4 & 14.3 & 2.0 & 1.9 \\
\hline $14 \mathrm{PW}$ & 28 & 36.6 & 70.9 & 1.1 & 3.8 & 1.6 & 69.9 & 0.8 & 8 & 44.8 & 19.6 & 0.6 & 5.7 \\
\hline $15 \mathrm{PW}$ & 48 & 73.2 & 70.9 & 18.4 & 7.0 & 3.1 & 181.4 & 0.9 & 244 & 136.8 & 32.9 & 9.2 & 2.6 \\
\hline $16 \mathrm{PW}$ & 24 & 12.2 & 88.6 & 0.9 & 4.2 & 1.7 & 11.8 & 0.8 & 72 & 26.4 & 17.3 & 0.8 & 1.5 \\
\hline 17PW & 36 & 158.6 & 70.9 & 1.6 & 8 & 1.6 & 199.5 & 1.1 & 92 & 43.2 & 130.4 & 1.6 & 3.9 \\
\hline 18RW & 24 & 36.6 & 88.6 & 0.9 & 4.5 & 5.1 & 214.3 & 0.8 & 20 & 4.8 & 105.2 & 42.7 & 1.0 \\
\hline 19RW & 24 & 97.6 & 70.9 & 14.3 & 1.8 & 4.8 & 101 & 0.8 & 16 & 4.8 & 38.6 & 2.2 & 1.4 \\
\hline $\begin{array}{c}\text { Sample } \\
\text { ID }\end{array}$ & $\begin{array}{c}\mathrm{V} \\
\text { (ppm) }\end{array}$ & $\begin{array}{c}\mathrm{Cr} \\
(\mathrm{ppm})\end{array}$ & $\begin{array}{c}\mathrm{Cu} \\
(\mathrm{ppm})\end{array}$ & $\begin{array}{c}\mathrm{Ge} \\
(\mathrm{ppm})\end{array}$ & $\begin{array}{c}\text { As } \\
(\mathrm{ppm})\end{array}$ & $\begin{array}{c}\mathbf{R b} \\
(\mathrm{ppm})\end{array}$ & $\begin{array}{c}\mathrm{Sr} \\
(\mathrm{ppm})\end{array}$ & $\begin{array}{c}\mathrm{Nb} \\
(\mathrm{ppm})\end{array}$ & $\begin{array}{c}\text { Mo } \\
(\mathrm{ppm})\end{array}$ & $\begin{array}{c}\text { Hf } \\
(\mathrm{ppm})\end{array}$ & & $\begin{array}{l}\text { Ta } \\
\text { pm) }\end{array}$ & $\begin{array}{c}\mathrm{W} \\
(\mathrm{ppm})\end{array}$ \\
\hline 1RW & 1.4 & 2.3 & 1.3 & 0.01 & 0.71 & 1.33 & 75.44 & 0.03 & 0.41 & 0.02 & & 002 & 0.05 \\
\hline 2RW & 0.9 & 2.5 & 1.4 & 0.01 & 0.66 & 1.39 & 54.64 & 0.03 & 0.20 & 0.01 & & 001 & 0.03 \\
\hline 3RW & 0.9 & 2.7 & 1.4 & 0.02 & 0.95 & 1.42 & 101 & 0.03 & 0.38 & 0.01 & & 001 & 0.03 \\
\hline 4SW & 1.4 & 1.3 & 1.2 & 0.03 & 1.84 & 5.51 & 81.75 & 0.04 & 0.20 & 0.04 & & 003 & 0.03 \\
\hline 5RW & 0.9 & 1.1 & 0.4 & 0.02 & 0.58 & 0.86 & 814.5 & 0.03 & 0.13 & 0.01 & & 001 & 0.02 \\
\hline $6 \mathrm{RW}$ & 4.6 & 1.7 & 6.7 & 0.07 & 1.01 & 1.33 & 623.7 & 0.03 & 0.20 & 0.04 & & 008 & 0.03 \\
\hline 7RW & 1.4 & 1.1 & 1.5 & 0.03 & 1.20 & 1.28 & 877.9 & 0.03 & 0.26 & 0.06 & & 011 & 0.03 \\
\hline 8LW & 1.2 & 0.9 & 1.7 & 0.05 & 0.81 & 1.85 & 57.0 & 0.03 & 0.09 & 0.03 & & 002 & 0.03 \\
\hline 9LW & 0.8 & 0.9 & 0.3 & 0.04 & 0.61 & 1.42 & 81.07 & 0.03 & 0.08 & 0.02 & & 001 & 0.02 \\
\hline $10 \mathrm{LW}$ & 0.9 & 0.8 & 0.2 & 0.03 & 0.65 & 1.52 & 65.67 & 0.03 & 0.02 & 0.07 & & 004 & 0.03 \\
\hline $11 \mathrm{LW}$ & 4.7 & 1.7 & 7.7 & 0.12 & 1.28 & 2.29 & 750.7 & 0.03 & 0.36 & 0.05 & & 002 & 0.03 \\
\hline $12 \mathrm{LW}$ & 0.8 & 0.8 & 0.4 & 0.04 & 0.72 & 1.58 & 67.47 & 0.03 & 0.04 & 0.01 & & 001 & 0.02 \\
\hline 13RW & 1.5 & 0.7 & 1.6 & 0.04 & 1.83 & 1.76 & 86.46 & 0.04 & 0.31 & 0.10 & & 034 & 0.05 \\
\hline 14PW & 7.3 & 3.1 & 10.0 & 0.15 & 1.16 & 3.64 & 737.8 & 0.04 & 0.22 & 0.05 & & 003 & 0.0 \\
\hline 15PW & 7.5 & 1.2 & 10.2 & 0.06 & 2.38 & 5.89 & 538.1 & 0.04 & 0.77 & 0.11 & & 011 & 0.05 \\
\hline 16PW & 5.7 & 1.6 & 9.2 & 0.05 & 1.38 & 2.92 & 582.6 & 0.04 & 0.76 & 0.08 & & 004 & 0.04 \\
\hline 17PW & 7.7 & 4.9 & 11.2 & 0.15 & 1.48 & 2.24 & 637.5 & 0.04 & 0.37 & 0.06 & & 004 & 0.03 \\
\hline 18RW & 1.0 & 0.4 & 0.8 & 0.02 & 0.87 & 1.09 & 91.93 & 0.03 & 0.10 & 0.01 & & 001 & 0.02 \\
\hline 19RW & 4.8 & 1.1 & 8.1 & 0.06 & 1.09 & 1.23 & 496.3 & 0.03 & 0.39 & 0.03 & & 002 & 0.02 \\
\hline
\end{tabular}


Table 2. Statistical information of hydrochemical data of water samples collected from wetlands of the Manipur valley, NE India.

\begin{tabular}{|c|c|c|c|c|c|c|}
\hline Parameters & Mean & SD & Min & Max & Skewness & Median \\
\hline $\mathrm{pH}$ & 7.3 & 0.69 & 6.2 & 9.3 & 0.89 & 7.3 \\
\hline TDS & 84.63 & 49.12 & 28 & 256 & 2.46 & 77 \\
\hline ORP & -29.42 & 36.78 & -129 & 35 & -0.57 & -30 \\
\hline $\mathrm{CO}_{3}$ & 29.47 & 10.17 & 16 & 60 & 1.87 & 24 \\
\hline $\mathrm{HCO}_{3}$ & 55.22 & 34.83 & 12.2 & 158.6 & 1.43 & 70.9 \\
\hline $\mathrm{Cl}$ & 76.49 & 22.15 & 35.45 & 141.8 & 1.23 & 70.9 \\
\hline $\mathrm{SO}_{4}$ & 7.616 & 10.89 & 0.1 & 46.8 & 2.83 & 4.3 \\
\hline $\mathrm{PO}_{4}$ & 3.00 & 1.88 & 1.1 & 8 & 1.55 & 2.6 \\
\hline $\mathrm{H}_{4} \mathrm{SiO}_{4}$ & 121.52 & 86.58 & 5.0 & 264.4 & 0.08 & 111.7 \\
\hline $\mathrm{Na}$ & 26.9 & 33.29 & 8.57 & 130.44 & 2.60 & 14.64 \\
\hline K & 4.31 & 9.54 & 0.6 & 42.72 & 4.02 & 1.48 \\
\hline F & 0.85 & 0.069 & 0.79 & 1.068 & 2.57 & 0.84 \\
\hline $\mathrm{Ca}$ & 52.84 & 64.67 & 8.0 & 244 & 1.80 & 16 \\
\hline $\mathrm{Mg}$ & 29.16 & 34.74 & 2.4 & 136.8 & 1.88 & 16.8 \\
\hline $\mathrm{Ti}$ & 1.85 & 1.99 & 0.259 & 7.917 & 2.16 & 1.17 \\
\hline V & 2.92 & 2.59 & 0.767 & 7.682 & 0.89 & 1.38 \\
\hline $\mathrm{Cr}$ & 1.60 & 1.08 & 0.431 & 4.902 & 1.79 & 1.16 \\
\hline $\mathrm{Cu}$ & 3.96 & 4.087 & 0.225 & 11.21 & 0.72 & 1.53 \\
\hline Ge & 0.05 & 0.04 & 0.006 & 0.154 & 1.49 & 0.04 \\
\hline As & 1.12 & 0.49 & 0.58 & 2.378 & 1.13 & 1.01 \\
\hline $\mathrm{Rb}$ & 2.13 & 1.42 & 0.862 & 5.894 & 1.89 & 1.52 \\
\hline $\mathrm{Sr}$ & 359.03 & 318.31 & 54.64 & 877.9 & 0.35 & 101 \\
\hline $\mathrm{Nb}$ & 0.03 & 0.01 & 0.025 & 0.043 & 0.40 & 0.03 \\
\hline Mo & 0.28 & 0.21 & 0.022 & 0.775 & 1.21 & 0.22 \\
\hline Hf & 0.04 & 0.03 & 0.004 & 0.114 & 0.89 & 0.04 \\
\hline $\mathrm{Ta}$ & 0.01 & 0.01 & -0.001 & 0.034 & 3.23 & 0.002 \\
\hline W & 0.03 & 0.01 & 0.018 & 0.054 & 1.03 & 0.03 \\
\hline
\end{tabular}

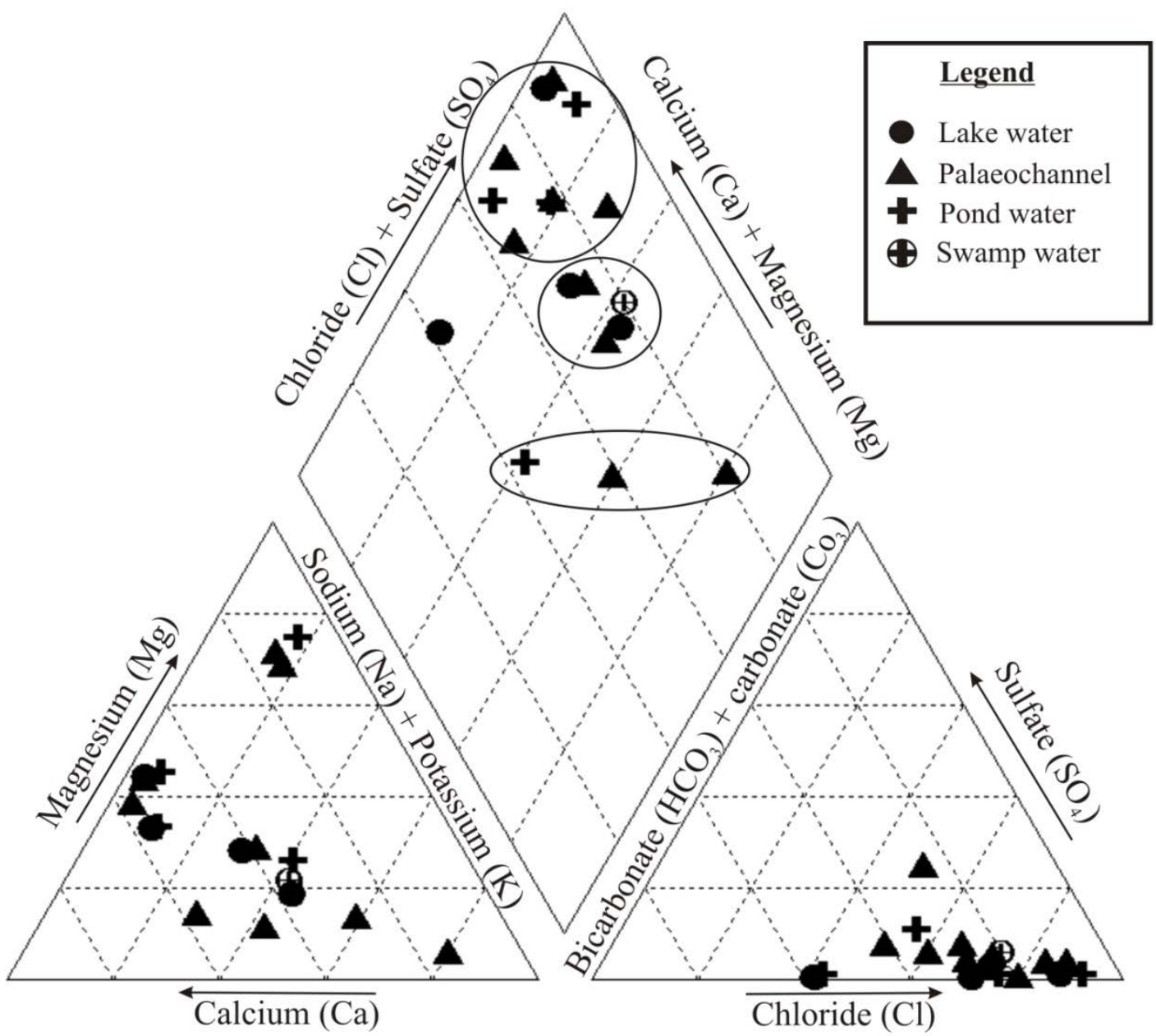

Figure 2. Trilinear diagram showing types of hydrochemical facies in the water samples collected from wetlands of Manipur Valley, NE India 


\subsection{Chemical Evolution Water}

As evident from the Trilinear diagram [8] (Figure 2), the wetlands show three types of hydrochemical facies; (i) $\mathrm{Cl}-\mathrm{SO}_{4}-\mathrm{Ca}-\mathrm{Mg}$, (ii) $\mathrm{Ca}-\mathrm{Mg}-\mathrm{Cl}-\mathrm{SO}_{4}-\mathrm{Na}$ and (iii) $\mathrm{HCO}_{3}-$ $\mathrm{Na}-\mathrm{Ca}-\mathrm{SO}_{4}$. Out of these, the first and second facies are the dominant types indicating ion exchange process in the initial and intermediate stages of chemical evolution in these waters. Further, it implies ions dissolution in these waters is originated from rocks like limestone, dolomite, sandstone and shales.

\subsection{The Flora and Fauna}

Wetlands in the valley render habitat for flora and fauna. Commonly found fishes in the ponds are Clarias batrachus ( Ngakra), Heteropneustes fossilis (Ngachik), Notopterus chitala (Ngapai), Channa striatus (Ngamu-porom), Channa marulius (Porom), Channa gachua (Meiteingamu), Anabas testudineus (Ukabi), Colisa faciata (Ngapema), Monopterus albus (Ngaprum), Lepidocephalus guntea (Ngakrijou), Ambassis nama (Ngamhai), Amblypharyngodon mola (Mukanga), Macrobrachium lamerii (Khajing), Puntius ticto (Ngakha), Tilapia mossambica (Tunghanbi), Acanthopthalmus pangia (Nganap). Generally, floras in the wetlands are dominated by phytoplankton (Chlorophyceae sp., Bacillariophyceae sp., Zoomastigophora sp.), zooplankton (Cyclops sp., Chydorous sp., Asplanchna sp.) and macrovegetation. Macrovegetation consists of many species of seasonal, annual and perennial plants. Commonly found genera are Centralla asiatica, Chara sp., Elipta alba, Euryale ferox, Marshilea quadrifolia and Scirpus sp. [9]. Common insects habitat in ponds are Grasshopper sp. Corixa sp. (water boatman), Notonecta sp. (Backswimmer), Gerris sp. (water strider), Cybister sp. (diving beetle), Sympetrum sp. (dragonfly), Nepa sp., Mantis sp. [10].

\subsection{The Present State}

In the modern Manipuri society, these wetlands are owned by individual or by community or by state or central government. Presently, they are used mainly for domestic and irrigation water supply, for fish production, for small industry like brick field, for recreation and for ritual activities. Urban sprawling studies based on Landsat imageries for the years 1977, 1990, 2000 and 2010 show that the area of Imphal city has expanded from $4.29 \mathrm{~km}^{2}$ in 1977 to $34.2 \mathrm{~km}^{2}$ in 2010 [11]. This urbanization, massive increase in population (from 22.94 lakhs in 2001 census to 27.21 lakhs in 2011 census) and shrinkage of wetlands cause reduction in the number of pre-existing ponds. Also, mismanagement and negligence on the treatment of domestic solid waste deteriorate the quality of pond water. Based on the database generated from Google image 2017 Digital Globe, geographic coordinates and areas of some of the major ponds in the Imphal valley is tabulated in Table 3. Based on recent studies on water quality of ponds carried out by Manipur Pollution Control Board revealed that pond water of the valley has high level of bacteria content (1000 to $5000 \mathrm{MPN})$. Artificial eutrophication coupled with climate change as it reduces oxygen level and increases acidic level in the pond waters may also lead to the extinction of some fish species. International Union for Conservation of Nature (IUCN) announced seven fish species of Ngakha Meingangbi (Puntius manipurensis), Ngatup (Schistura Kanjupkhulensis), Ngatup makhal ama (Schistura minutes), Ngatup manba nga (Schistura reticula), Ching-ngakra (Pterocryptis barakensis), ChingUkabi (Badis tuivaiei) and Nung-nga (Psilorhynchus microphthalamus) as 'endangered freshwater fish species' recently.

Table 3. Geographic information of historically significant ponds presently locating in the Manipur valley, NE India

\begin{tabular}{|c|c|c|c|}
\hline $\begin{array}{l}\text { Sl. } \\
\text { No. }\end{array}$ & Name of the ponds & $\begin{array}{l}\text { Geographic } \\
\text { coordinates }\end{array}$ & $\begin{array}{c}\text { Area } \\
\left(\mathrm{m}^{2}\right)\end{array}$ \\
\hline 1 & Ningthem pukhri & $\begin{array}{l}24^{\circ} 47^{\prime} 56^{\prime \prime N} \\
93^{\circ} 57^{\prime} 16^{\prime \prime} \mathrm{E}\end{array}$ & 4031 \\
\hline 2 & Naharup Awang leikai Road pukhri & $\begin{array}{l}24^{\circ} 47^{\prime} 26^{\prime \prime N} \\
93^{\circ} 58^{\prime} 15^{\prime \prime} \mathrm{E}\end{array}$ & 1073 \\
\hline 3 & Kongba Uchekon Khunou pukhri & $\begin{array}{l}24^{\circ} 46^{\prime} 19^{\prime \prime N} \\
93^{\circ} 58^{\prime} 13^{\prime \prime} \mathrm{E}\end{array}$ & 916 \\
\hline 4 & Manipur University pukhri & $\begin{array}{l}24^{\circ} 44^{\prime} 56^{\prime \prime N} \\
93^{\circ} 55^{\prime} 43^{\prime \prime} \mathrm{E}\end{array}$ & 880 \\
\hline 5 & Patsoi pukhri & $\begin{array}{l}24^{\circ} 47^{\prime} 07^{\prime \prime N} \\
93^{\circ} 52^{\prime} 42^{\prime \prime} \mathrm{E}\end{array}$ & 746 \\
\hline 6 & Sagolband pukhri & $\begin{array}{l}24^{\circ} 47^{\prime} 45^{\prime \prime N} \\
93^{\circ} 54^{\prime} 03^{\prime \prime} \mathrm{E}\end{array}$ & 742 \\
\hline 7 & Kongba pukhri & $\begin{array}{l}24^{\circ} 45^{\prime} 30^{\prime \prime N} \\
93^{\circ} 57^{\prime} 46^{\prime \prime} \mathrm{E}\end{array}$ & 736 \\
\hline 8 & Heinoupok pukhri & $\begin{array}{l}24^{\circ} 47^{\prime} 33^{\prime \prime N} \\
93^{\circ} 54^{\prime} 03^{\prime \prime} \mathrm{E}\end{array}$ & 663 \\
\hline 9 & Khongman pukhri & $\begin{array}{l}24^{\circ} 45^{\prime} 30^{\prime \prime N} \\
93^{\circ} 57^{\prime} 46^{\prime \prime} \mathrm{E}\end{array}$ & 639 \\
\hline 10 & Wakha pukhri & $\begin{array}{l}24^{\circ} 48^{\prime} 00^{\prime \prime N} \\
93^{\circ} 59^{\prime} 01^{\prime \prime} \mathrm{E}\end{array}$ & 607 \\
\hline 11 & Makha leikai pukhri & $\begin{array}{l}24^{\circ} 46^{\prime} 19^{\prime \prime N} \\
93^{\circ} 58^{\prime} 13^{\prime \prime} \mathrm{E}\end{array}$ & 512 \\
\hline
\end{tabular}

\section{Conclusions}

The hydrochemical analysis of wetland samples of Manipur valley has carried out. Based on these parameters, the qualities of these waters are found fresh and suitable for human consumption as well as flora and fauna ecology. The hydrocheical facies indicates the water is derived from geogenic weathering of rocks like shales, limestones, sandstones etc. The chemical evolution of these waters belongs to initial and intermediate stages further implying meteoric and fresh quality. However, these wetlands are degrading in terms of its quantity in the last few decades due rapid urbanization and increase in population. Many flora and fauna species have already lost as these wetlands are shrinking. Therefore, it is necessary to avoid demolishing wetlands and support to rejuvenate and maintain all the existing wetland bodies at the best possible ways. These forms of water bodies must be highly regarded and respected considering the age-old relationship it bears.

\section{Acknowledgements}

The authors are grateful to the director, IIT Powai, India for allowing us to carry out laboratory analysis of samples. Special thanks to Prof. S. Balakrishnan and Dr. Srinivasamoorthy of department of Earth Sciences, 
University of Pondicherry, India for their constant suggestions and guidance during the sample analysis at the University.

\section{References}

[1] MRSAC (2012), Manipur remote sensing application centre, Landuse/landcover map, Gov. of Manipur.

[2] Keddy, P.A., Wetland Ecology: Principles and Conservation (2nd edition), Cambridge University Press, Cambridge, UK, 2010, pp. 497.

[3] Laisam, K.S., Encyclopedia of Manipur, Vol 3, Gyan Publishing House, 2011, pp. 1175.

[4] Soibam, I., Structural and tectonic analysis of Manipur with special reference to evolution of the Imphal Valley. Ph.D thesis, 1998, pp 235, Manipur University, India, http://hd.handle.net/10603/39464.

[5] Ranga Rao., A., Geology and hydrocarbon potentials of a part of Assam-Arakan basin and its adjoining region. Symposium petroliferous basins of India, 1983, pp. 127-158.
[6] Kshetrimayum, K.S. and Thokchom, L. (2017) Hydrogeochemical approach to evaluate the occurrence and source of salinization in the shallow aquifers of the eastern Imphal valley in the IndoMyanmar Range of Northeastern India. Environmental Earth Sciences, 76:714-732, 2017.

[7] CGWB., Central Ground Water Board, Groundwater information booklet Imphal west district, Manipur, Technical report series D, No. 28/2009-10, 2009.

[8] Hill, R.A., Geochemical patterns in Coachella Valley, California. Trans Am Geophys Union 21:46-53, 1940.

[9] Dasgupta, M., A study on the Limnology of two freshwater ponds of Imphal, Manipur. Indian Journal of Fish, vol. 40(4), pp. 256261, 1993.

[10] Soram, J.S., Gurumayum, S., and Abujam, S.K., Diversity of fish and insect fauna of 'Sidizii' A hill stream, Manipur, International journal of science and nature, vol. 3(4), pp. 836-841, 2012.

[11] Khan, M.A., Ahmad, S., and Matloob, H., Landuse pattern, house design and health in Imphal valley, The NEHU journal, Vol. X (1), pp.81-92, 2012. 